\title{
A PROPOS DE L'ElEVAgE EN CAPTIVITE DE JEUNES PHOQUES (PHOCA VITULINA LINNAEUS, 1758)
}

\author{
par \\ AGATHA GIJZEN \\ Société Royale de Zoologie d'Anvers
}

La naissance de jeunes phoques a lieu dans nos régions, en général au cours des mois de juin-juillet. C'est alors qu'après une gestation d'environ 11 mois, le jeune vient au jour, mesurant $80-85 \mathrm{~cm}$ de longueur et pesant de $12 \mathrm{à} 20 \mathrm{~kg}$; il est la plupart du temps déjà recouvert du deuxième pelage brillant aux reflets argentés. Le pelage embryonal du début (lanugo) est de façon générale rejeté avant la naissance, mais parfois aussi pendant le processus de la mise bas; on le trouve alors sur la côte avec l'arrière-faix. La mère nourrit son rejeton (pup) à terre, jusqu'à ce qu'il soit devenu dodu ( \pm 4 semaines), après quoi il est abondonné à lui-même (1). Après quelques jours ou quelques semaines de difficultés, le jeune a finalement appris à s'alimenter et se développe en ,yearling”. Jusqu'ici tout est normal et naturel.

Mais il arrive de temps en temps que naissent des jumeaux. Comme chez les phoques c'est la mère qui suit le jeune et non le jeune qui suit la mère, il en résulte régulièrement qu'un des jumeaux reste en arrière sans assistance maternelle. Vu que la mère suit le jeune qui peut-être criait le plus fort ou que pour telle ou telle raison elle préféra, il se fait que le deuxième jeune reste sans aide et affamé, appelant instinctivement l'aide maternelle jusqu'à ce qu'il meure d'épuisement; le nom de „Heuler-huiler” (criard) utilisé internationalement pour désigner le jeune abandonné, est donc bien choisi.

Il peut arriver, naturellement, que la mère meure par exemple d'un coup de feu ou pour une autre cause; dans ce cas le rejeton qu'elle escortait reste seul, abandonné et termine son existence comme „criard" épuisé.

Ce sont en général ces „criards”, ces jeunes abandonnés (2) et désemparés, que l'on trouve sur la côte, qui sont receuillis par ceux qui les ont pris à charge et qui pour la plupart, finissent après plus ou moins d'avatars par échouer dans un jardin zoologique.
Ceci n'est pas seulement le cas pour Anvers, mais aussi pour Rotterdam, Amsterdam, Bremerhaven, soit pour tous les jardins zoologiques qui ne sont pas trop éloignés du littoral.

Nous savons tous instinctivement comment soigner un jeune animal et, c'est ainsi qu'on a rapidement recours au biberon rempli de lait additionné d'eau et dont on s'efforce de faire entrer la tétine dans le petit museau du jeune phoque. S'il s'agit d'un très jeune animal (à constater d'après l'ombilic; le cordon ombilical tombe après 4-5 jours; la cicatrice est fermée en 7 jours), il y a peu de résistance. Un jeune un peu plus âgé est parfois agressif et, je sais d'expérience personelle que les petites dents sont très acérées et que leur morsure a souvent pour suite une très désagréable infection. S'il s'agit d'un animal docile, on arrivera bien à placer la tétine de façon idoine dans le bec. Mais cela ne veut pas dire que le jeune phoque se met à têter immédiatement! Les tétins du phoque sont notamment très petits et comme enfouis dans la paroi ventrale, d'où chez le jeune, une façon toute différente de saisir la tétine du biberon, fabriquée d'après modèle humain. Et voici que les difficultés commencent. Après de nombreux essais infructueux, on arrive enfin à faire pénétrer la tétine et à faire têter l'animal. En laissant couler goutte à goutte le lait sur la main et en le laissant sucer par l'animal, on arrive parfois à des résultats. Mais tout cela ne veut pas dire que nous soyons occupés à élever un jeune phoque. Car tout ce qu'il prend ainsi est tout au plus suffisant pour maintenir ses humeurs, mais totalement insuffisant pour le faire grandir. Le lait de phoque contient en effet de 9,7 à 10,4\% d'albumine (soit 3 fois plus que le lait de vache), et rien moins que $42 \%$ de graisse (12 à 13 fois autant que le lait de vache). D'autre part, d'après l'expérience avec des enfants humains, ce lait de vache est encore étendu d'eau! De nombreux jardins 
zoologiques eurent déjà à faire face à ce problème et toujours ils eurent à enregistrer à plus ou moins longue échéance, des échecs.

Mème lorsqu'on était arrivé à faire accepter par le jeune phoque de lait renforcé de blanc d'oeuf et d'huile de foie de morue (ce qui constitue un aliment à peu près aussi nutritif que le lait de phoque) la transition ultérieure, à une alimentation de poisson restait l'obstacle critique.

Je réussis en 1947, au Jardin Zoologique d'Anvers, à élever un petit phoque abandonné, soit un „criard”, qu'on nous avait remis, et à en faire un robuste jeune individu, en l'alimentant à l'aide du lait renforcé précité, que l'animal suçait de ma main qui entourait la tétine (3). Je nommai l'animal Alpha, sans me douter qu'au cours des années suivantes, presque tout un alphabet de phoques dont l'élévage avait réussi, viendrait s'ajouter à l'Alpha en question! Il fut prudemment habitué à accepter d'abord des filets de merlan, plus tard des merlans entiers mais dont, pendant un certain temps, on avait enlevé la tête.

Peu de temps après que l'animal eut été amené tout à fait à une alimentation normale, il perdit l'appétit et fut sujet à des crampes. Il mourut \pm 36 heures après le début de la maladie. L'autopsie établit que le décès était dû à une gastro-entérite.

On se demande quelle peut être la cause d'une telle affection. Une infection? Des troubles digestifs à la suite d'une alimentation impropre? En tout cas, il apparut sans plus que la méthode utilisée n'était pas ce qu'il fallait.

Nous décidâmes l'année suivante d'essayer de nouveau de trouver un bon système. Par hasard il se fit qu'on nous offrit (le 4 aout 1948) un très jeune couple qui avait déjà été amené à s'alimenter de poisson. Avec Bobby, le mâle, on n'eut aucune difficulté; il est encore toujours au Zoo et est donc âgé présentement de 13 ans. La femelle mourut le 2 septembre, soit après un séjour d'environ un mois au Zoo, des suites d'une gastrite et d'une pneumonie. D'autres exemplaires reçus la même année, moururent à bref délai. La bonne solution de l'élevage des jeunes phoques en captivité restait donc toujours à trouver.

Nous décidâmes finalement d'essayer d'amener le plus vite possible les jeunes phoques à une alimentation de poisson. A cet effet nous préparîemes une purée de filets de merlan broyés, allongée de lait et d'huile de foie de morue. De cette façon la masse épaisse devenait fluide et sa teneur en matières grasses était augmentée. Pour chaque animal on réserva un entonnoir auquel était ajusté un tuyau en matière plastique qu'on introduisait dans le gosier du bébéphoque. Une quantité du purée, bien déterminé pour chaque animal, fut placée dans l'entonnoir et poussée dans le tuyau introduit dans le gosier du pup, de façon qu'elle parvint directement dans l'estomac de celui-ci. On évitait ainsi la si néfaste déglutition défectueuse et on savait exactement la quantité de nourriture ingurgitée, vu que, de cette façon, rien ne se perdait. C'était déjà toute une affaire avec des animaux dociles; mais lorsqu'il s'agissait de ,rebelles" c'était un tour de force. Il fallait alors en plus du gardiensoigneur au moins deux hommes pour tenir un petit animal pesant quelque 20 kilos! Le gardien chargé de l'alimentation imagina alors de remplacer l'entonnoir par une seringue injectante comme on en utilise en ménage - la vérité nous oblige à reconnaître qu'il „emprunta" l'appareil à l'armoire aux ustensiles de sa femme. La purée nutritive était désormais refoulée sous pression directement dans l'estomac des jeunes phoques, par la seringue injectante. Il suffisait d'un seul homme pour maneuvrer celle-ci tandis qu'un autre maintenait l'animal à gâver (4). Plus tard nos techniciens fabriquèrent une seringue en métal car celle, qui fut utilisée au début était en matière plastique et trop fragile pour résister aux chocs résultant des mouvements des animaux turbulents, et à la pression exercée sur la purée de consistance relativement forte. Lorsque cette méthode se fut avérée favorable, notre gardien-inventeur imagina et confectionna un appareil basé sur le même principe mais perfectionné et qui ne nécessite que la présence d'un seul homme: une pompe montée en bois pourvue d'un tube métallique, d'un piston en bois et d'un tuyau souple en matière plastique $(5,6,7)$.

Nous pouvons affirmer à présent que grâce au zèle et à l'ingénosité inventive de ce gardien, quelconque jeune phoque, reçu en bonne condition et non trop parasité, ni blessé, a toute les chances de grandir et de prospérer. Evidemment, si les jeunes animaux sont fort meurtris ou épuisés, ou encore s'ils sont atteints d'une inflammation ombilicale ou s'ils ont avalé trop de résidus d'huile ou de pétrole, il n'y a guère de chance, même avec cette méthode, de vaincre les difficultés résultant de leur piètre état physique.

En marge de la méthode exposée ci-dessus il y a encore d'autres possibilités de mener à bonne fin l'élevage des jeunes phoques. Dans les „Tiergrotten” de Bremerhaven, où en raison de la situation, on a acquis une grande expérience en la matière, on gâve à la main, les jeunes phoques, avec des filets de poisson. Le bon résultat obtenu de cette manière avec 19 sur 27 pups soumis à ce régime, prouve qu'il y a moyen de cette façon d'assurer leur élevage.

L'administration d'une préparation spéciale, utilisée pour l'élevage de porcelets privés de mère, connue 

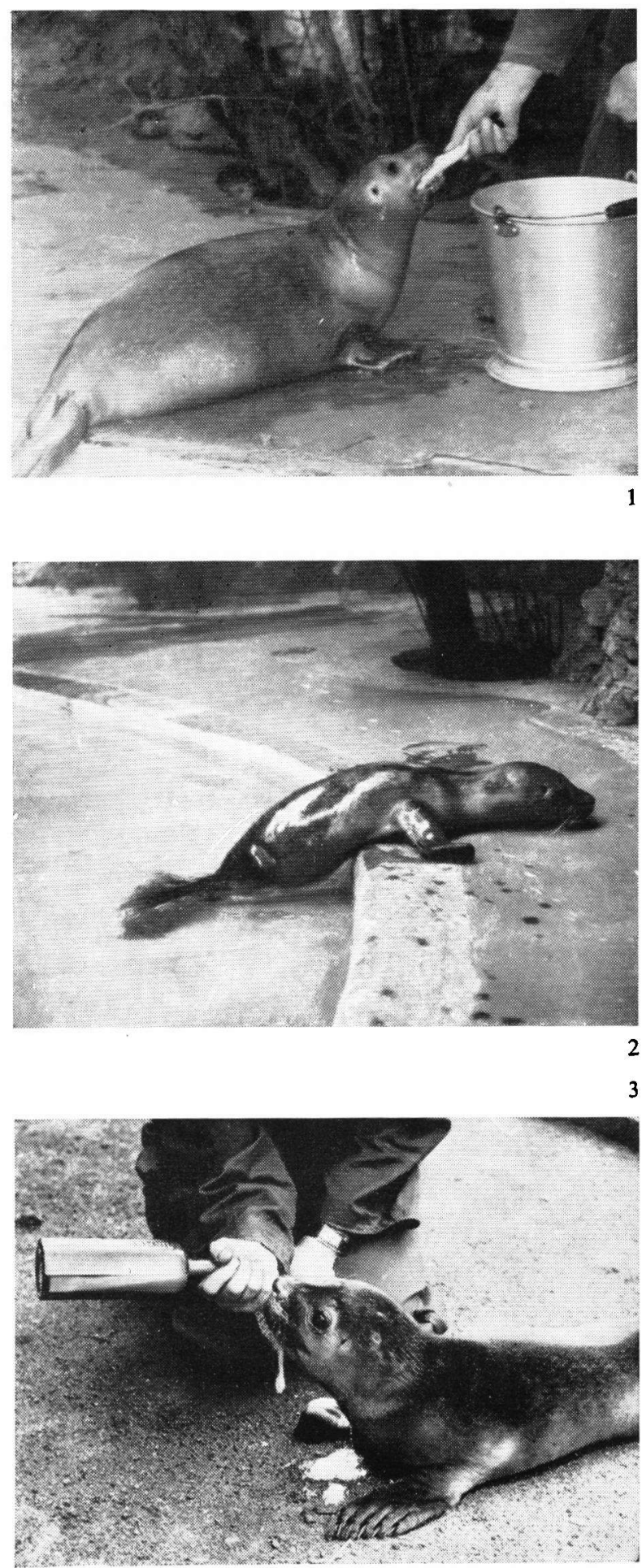

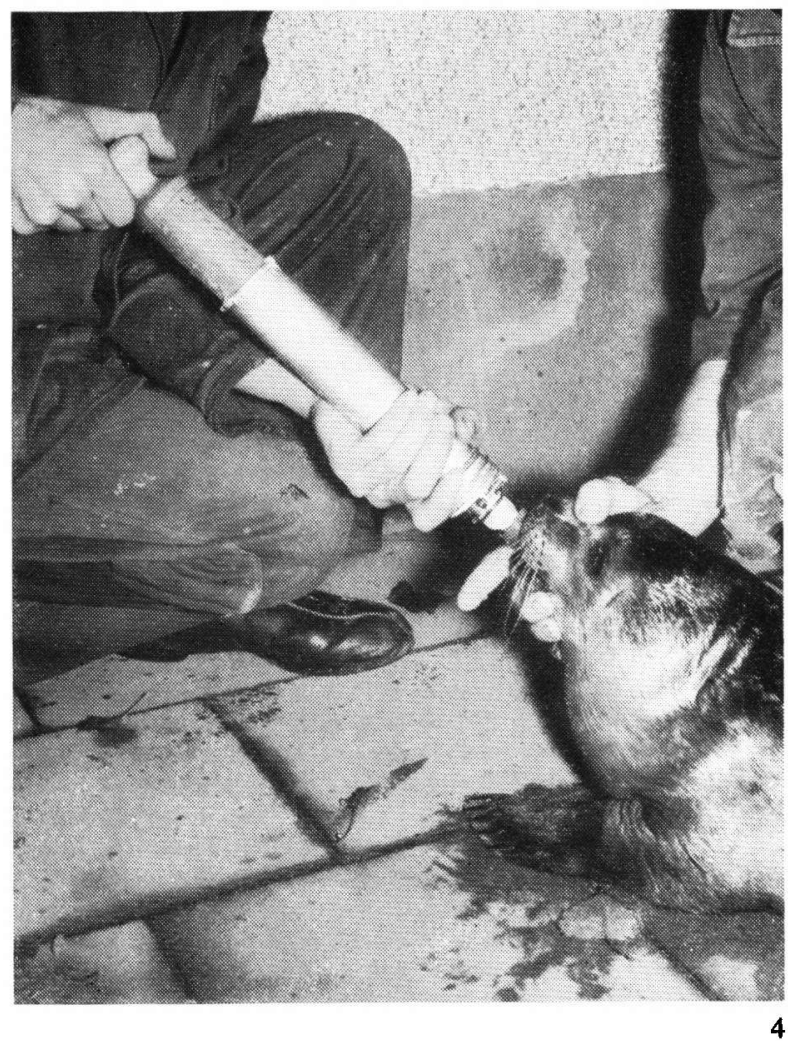

1. Jeune phoque, peu après le sevrage. L'animal est gras et dodu.

Photo V. Six

2. Jeune phoque recueilli abandonné (huiler $=$ criard).

Photo V. Six

3. Jeune phoque suçant la main de l'auteur.

Cliché Zoo Anvers

4. Le gardien bricola une espèce de grande seringue, à l'aide de la quelie on gâva les jeunes phoques. 
5. Jeune phoque avec l'appareil nourricier définitif.

Photo F. Nauwelaerts

6. Le tuyau, déjà rempli de nourriture, est introduit dans le gosier du jeune phoque.

Photo F. Nauwelaerts

7. La pompe à levier, permettant d'alimenter les jeunes phoques rapidement et sans autre assistance. Photo V. Six

8. Ah, que c'est bon!

Photo F. Nauwelaerts

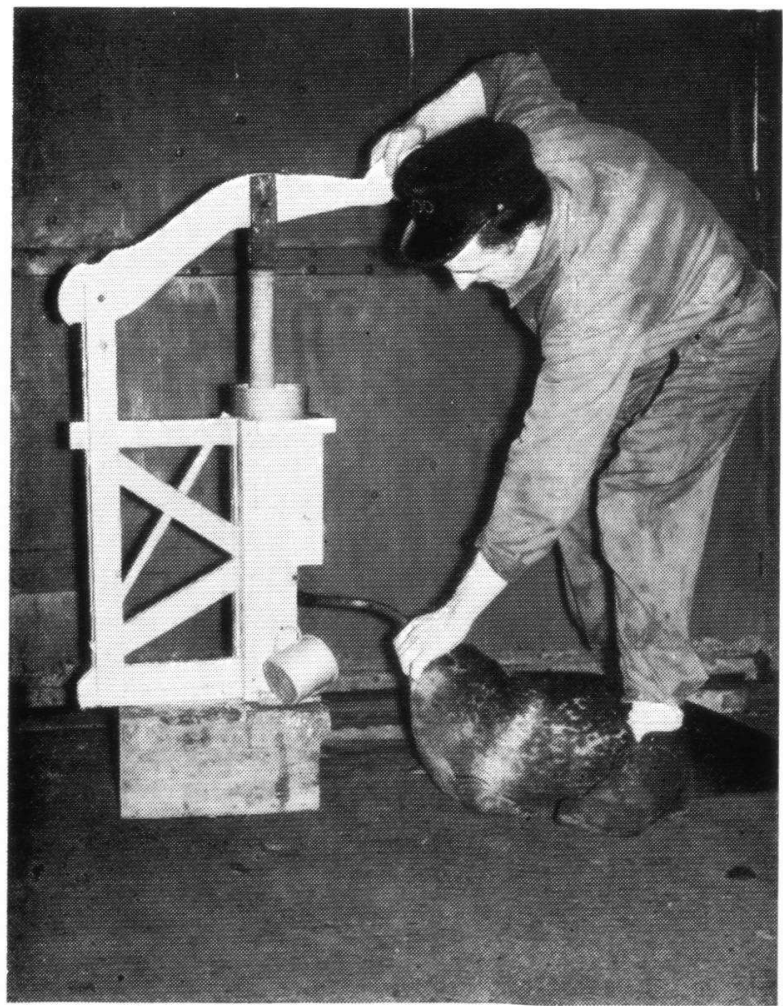

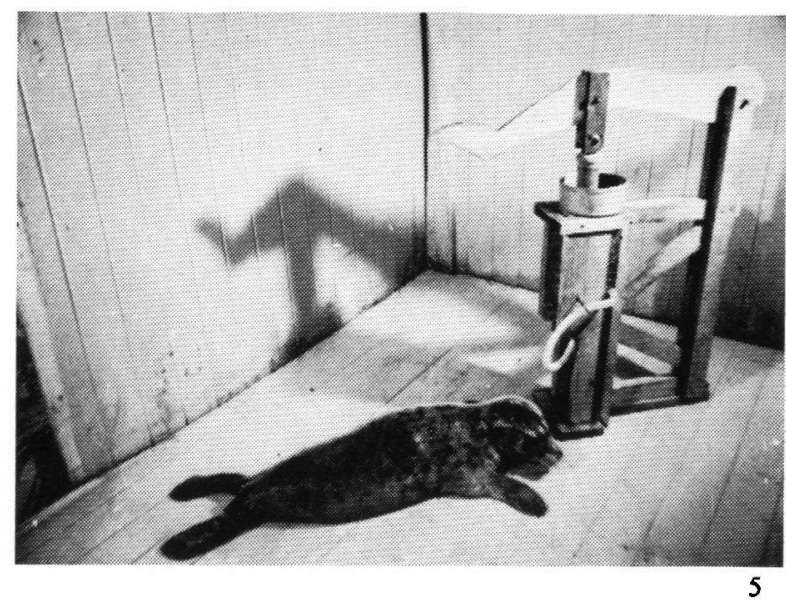

6

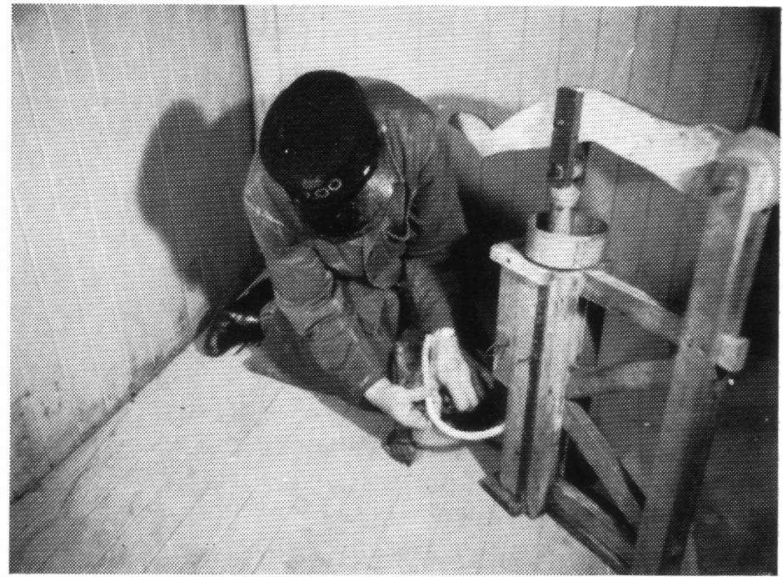

8

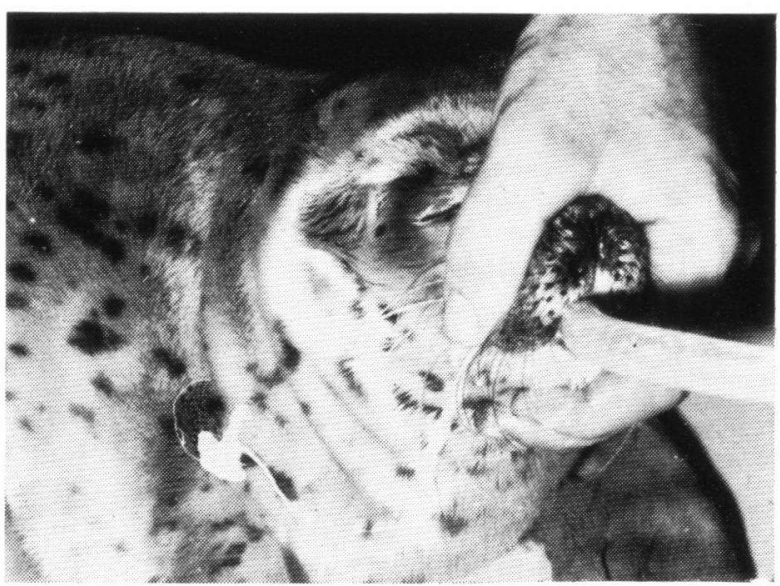


sous le nom de „Biggenmelk” et mise en vente en boîtes de fer blanc dans le commerce, donnerait au début - comme on me le confirma au Jardin Zoologique „Blijdorp” à Rotterdam - de bons résultats. Mais la transition à l'alimentation à l'aide de poisson reste encore toujours un moment critique et l'on n'a guère réussi jusqu'à présent à mener de la sorte, un jeune phoque jusqu'à l'état adulte.

Quoi qu'il en soit, je pense pouvoir affirmer que la façon de soigner et de nourrir utilisée à Anvers, et qui consiste à faire prendre au jeune phoque sans violence ni trop d'émotion, une quantité appropriée de nourriture - ensemble environ 3 merlans, 12 cuillères à café d'huile de foie de morue, un peu de lait et tous les deux jours un jaune d'oeuf - donne des chances raisonnables à un individu reçu en bonne santé, de croître et de prospérer (8).

\section{REFERENCES}

BERGH, W. van DEN, 1958: Succes et échec d'ordre zootechnique. Zoo XXIII, pp. 130-133.

Gijzen, A., 1956: Les Pinnipèdes. Zoo XXII, pp 2-36.
Morr, E., 1955: Der Seehund. Neue Brehm-Bücherei 145. Wittenberg Lutherstadt. A. Ziemsen Verlag.

Spector, W. S., 1956: Handbook of biological data. Philadelphia and London. W. B. Saunders Company. 